\title{
CONSUMO DE SUSTANCIAS PSICOACTIVAS EN ESTUDIANTES DE MAGISTERIO Y SUS CREENCIAS ACERCA DE LA EDUCACIÓN PREVENTIVA
}

\section{CONSUMPTION OF PSYCHOACTIVE SUBSTANCES IN STUDENT TEACHERS AND THEIR BELIEFS ABOUT PREVENTIVE EDUCATION}

\author{
MANUEL FERNÁNDEZ CRUZ ${ }^{1}$, JOSÉ GIJÓN PUERTA ${ }^{1}$ Y \\ EMILIO JESÚS LIZARTE SIMÓN ${ }^{1}$
}

Cómo referenciar este artículo/How to reference this article:

Fernández Cruz, M., Gijón Puerta, J. y Lizarte Simón, J. (2016). Consumo de sustancias psicoactivas en estudiantes de magisterio y sus creencias acerca de la educación preventiva [Consumption of psychoactive substances in student teachers and their beliefs about preventive education]. Acción Psicológica, 13(1), 67-78. http://dx.doi.org/10.5944/ap.13.1.17419

\section{Resumen}

La realización de programas escolares de prevención de consumo de drogas se ha demostrado eficaz en el control de hábitos de adicción en la población juvenil. El éxito de los programas preventivos descansa, en buena parte, en la capacitación de los docentes que los desarrollan y sus propias creencias sobre el consumo de drogas. Otro factor que puede estar relacionado con el éxito de los programas, es la experiencia del propio docente en el consumo de sustancias psicoactivas. En este artículo se explora la relación entre hábitos de consumo de tabaco, alcohol y drogas ilegales de futuros docentes (estudiantes

de magisterio de la Universidad de Granada) y las creencias falsas sobre prevención de la drogadicción en la escuela que mantienen.

Palabras clave: formación inicial docente; educación para la salud; dependencia; drogas.

Abstract
Conducting educational programs to prevent drug
use has proven effective in controlling addiction
habits among young people. The success of

\section{Abstract}

Correspondencia: Manuel Fernández Cruz. Departamento de Didáctica y Organización Escolar, Facultad de Ciencias de la Educación, Universidad de Granada, España.

Email:manuelfernandezcruz@gmail.com

${ }^{1}$ Universidad de Granada, España.

Recibido: 23 de marzo de 2016.

Aceptado: 12 de mayo de 2016. 
prevention programs rests largely on the training of teachers and their own beliefs about drug use. Another factor that may be related to the success of the programs is teacher's own experience in the use of psychoactive substances. In this article we explore the relationship between tobacco, alcohol and illegal drugs consumption habits of future teachers (education students at the University of Granada) and the false beliefs about drug prevention at schools they have.

Keywords: preservice teacher education; health education; dependency; drugs.

\section{Introducción. \\ Educación preventiva del consumo de sustancias psicoactivas}

\section{Prevalencia y efectos nocivos}

Una sustancia psicoactiva es aquella que se consume para modificar la forma en que las personas se sienten, piensan o comportan (ONU, 2002). La definición incluye al tabaco, al alcohol y a otras drogas. La gravedad y globalidad del problema del uso de estas sustancias psicoactivas en toda la población, pero de manera específica en edad escolar, se pone de manifiesto en los distintos informes elaborados en distintas regiones del mundo, lo que incluye el seguimiento bianual que se hace en España y en las distintas Comunidades Autónomas sobre patrones de consumo de tabaco, alcohol y otras drogas entre su población, con caracterización por intervalos de edad. En estos estudios se revela la alta prevalencia del consumo entre adolescentes y jóvenes. En Estados Unidos (CASA, 2011), más del $75 \%$ de los estudiantes de secundaria han consumido alguna vez tabaco, alcohol, marihuana o cocaína; más del $46 \%$ lo hacen con alta frecuencia; y más del $19 \%$ han tenido diferentes desórdenes clínicos causados por este consumo. Esas cifras son fácilmente transferibles a otras regiones desarrolladas del mundo. En España, si bien el consumo de tabaco se ha visto algo reducido con las leyes de 2005 y
2010 que han limitado su uso, las nuevas prácticas sociales que acompañan el consumo del alcohol como la del "botellón", junto a la aceptación social del consumo de algunas drogas ilegales como el cánnabis, han incrementado la presencia del uso de sustancias psicoactivas entre la juventud. El coste social de estas prácticas tanto a corto plazo, considerando la población joven, como en el plazo largo, considerando el conjunto de la población consumidora, es incalculable. Baste decir que el alcohol es una de las primeras causas prevenibles de morbilidad y mortalidad (Córdoba et al., 2012) aunque en la actualidad causa más de 2,5 millones anuales de muerte en el mundo. Por su parte, el tabaco constituye la primera causa de muerte prevenible en el mundo desarrollado. En cuanto al futuro de los jóvenes consumidores, Ringel, Ellickson y Collins (2007) han demostrado que los adolescentes consumidores de drogas van a ocupar, en el futuro, ocupaciones laborales de baja o muy baja calidad. Por su parte, en el estudio de Engberg y Morral (2006) se alerta del fracaso escolar asociado al consumo de drogas y sus efectos sociales y económicos posteriores.

\section{Programas de educación preventiva}

Ante la grave situación descrita, expertos y organismos internacionales plantean la educación preventiva sobre el consumo de tabaco, alcohol y otras drogas en la escuela, como una de las intervenciones más eficaces y rentables. La ONU recoge en su informe de 2002 el conocimiento y las buenas prácticas acumuladas por distintas organizaciones en educación preventiva. Sus recomendaciones se basan en los conceptos de participación activa de los jóvenes en el desarrollo de los programas preventivos; la eficacia probada de la actuación entre pares; y la aportación de soluciones diversas adaptadas a la realidad sociocultural que caracteriza el consumo de drogas en regiones diferentes. Para la ONU los principios básicos de la prevención son: (a) tener una base comunitaria con implicación de todos los agentes afectados; (b) abarcar con el programa, de manera global, a todo el conjunto de sustancias psicoactivas; (c) fijar en los propios jóvenes como destinatario del programa, los objetivos propuestos; (d) y priorizar las acciones para la evitación del inicio del consumo. 
En España, entre los documentos aportados por la Delegación del Gobierno para el Plan Nacional sobre Drogas, destacan los elaborados por la Addiction Research Foundation ARF (1991) que recomienda la prevención del consumo de drogas a través de la educación en actividades curriculares y extracurriculares en la escuela que han demostrado su eficacia en estudios como los de Harrison y Narayan (2003), Hoffmann (2006) o Dawkins, Wiliams y Guilbault (2006) quienes analizan la eficacia de la participación en actividades escolares deportivas, como medida preventiva del consumo; la intervención conjunta de recursos profesionales educativos y recursos terapéuticos bajo el liderazgo de los orientadores escolares es prioritaria para la prevención como lo han puesto de manifiesto trabajos como el de BurrowSanchez y Lopez (2009), Burrow-Sanchez, Call, Adolphson y Hawken (2009); la disposición de protocolos claros para abordar incidentes reales de consumo en la escuela que pueden incluir políticas escolares de tolerancia cero (APA, 2008) aplicadas con sabiduría y adaptación al contexto la priorización de estrategias de implicación de los alumnos en intervenciones de prevención con sus propios compañeros, implicación de las familias, e implicación del resto de la comunidad (Adams, Jason, Pokorny y Hunt, 2009); la alineación de directrices sobre formación, información, educación y orientación escolar (LaRusso, Romer y Selman, 2008) y, finalmente, la evaluación y seguimiento de objetivos propuestos y acciones desarrolladas y la formación del profesorado, junto a otro personal no docente, para liderar el proceso de prevención en la escuela (Fernández Cruz, 2015).

Tabla 1

Correlaciones entre consumidores, consumo de drogas y creencias erróneas

\begin{tabular}{|c|c|c|c|c|c|c|c|}
\hline \multirow{2}{*}{ Creencias } & & \multirow{2}{*}{ Sexo } & \multicolumn{5}{|c|}{ Consumo de } \\
\hline & & & $T$ & $\mathbf{A}$ & $\mathbf{H}$ & TP & TS \\
\hline $\begin{array}{l}\text { No se puede ayudar a los consumidores abusivos de } \\
\text { alcohol u otras drogas hasta que "toquen fondo" }\end{array}$ & $\begin{array}{l}r \\
\mathbf{N}\end{array}$ & $\begin{aligned}-.185^{*} \\
203\end{aligned}$ & & & & $\begin{array}{r}-.138 \\
203\end{array}$ & \\
\hline $\begin{array}{l}\text { Mientras un niño use solo "drogas blandas", como la } \\
\text { marihuana, o solamente alcohol y tabaco, es muy difícil }\end{array}$ & $r$ & $-.218^{n+}$ & .169 & -.138 & & & -.171 \\
\hline $\begin{array}{l}\text { que llegue a ser adicto, y por lo tanto no necesitará } \\
\text { ningún tipo de intervención }\end{array}$ & $\mathbf{N}$ & 203 & 202 & 203 & & & 203 \\
\hline $\begin{array}{l}\text { Lo que hacen los niños fuera de clase no es asunto de } \\
\text { la escuela }\end{array}$ & $\begin{array}{l}r \\
N\end{array}$ & $\begin{array}{r}-.197 \\
203\end{array}$ & & & & $\begin{array}{r}-.214 \\
203\end{array}$ & \\
\hline $\begin{array}{l}\text { Sólo los especialistas pueden impartir educación sobre } \\
\text { drogas }\end{array}$ & $\begin{array}{l}r \\
\mathrm{~N}\end{array}$ & & & & $\begin{array}{r}-.140 \\
203 \\
\end{array}$ & & \\
\hline
\end{tabular}

Nota. $\boldsymbol{r}$ : correlación de Pearson, ${ }^{*} p<.05 ;{ }^{* *} p<.01$. T: Tabaco, A: Alcohol, H: Hachís, TP: Tranquilizantes con prescripción, TS: tranquilizantes sin prescripción.

\section{Creencias erróneas en educación preventiva}

Los riesgos de la prevalencia del consumo, así como los efectos positivos de la educación preventiva en la escuela han sido ampliamente analizados. Contamos con estudios como el de Cox, Zhang, Johnson y Bender (2007) que correlacionan consumo de tabaco, alcohol y otras drogas con rendimiento académico; o indagan en factores de riesgo predictores del consumo de sustancias psicoactivas (Pisetsky, Chao, Dierker, May y StriegelMoore, 2008) como puede ser las actitudes escolares negativas, la inadaptación social y la baja autoestima (Bryant y Zimmerman, 2002).

Estos estudios enfatizan el papel del profesor durante la escolaridad obligatoria en la evitación del inicio del consumo y el desarrollo de una educación preventiva. Sin embargo, frecuentemente los profesores mantienen una serie de creencias erróneas sobre la educación preventiva que dificultan la eficacia de los programas. La ARF (1991) ya detectó algunas de estas creencias erróneas (ver tabla 1) que parecen mantenerse en el tiempo en las sucesivas generaciones de docentes.

Las creencias erróneas están relacionadas con el conocimiento que tienen los docentes sobre las drogas y su propia experiencia de consumo. La formación docente en educación preventiva, por tanto, debe considerar tanto la exhaustiva información sobre los efectos nocivos de las sustancias psicoactivas, como los factores predictivos de riesgo de consumo y los ejes de actuación que mejoran la eficacia de los programas. Para la formación docente en 
Tabla 2

Distribución de la Población y Muestra

\begin{tabular}{lcc}
\hline Titulación & Población & Muestra \\
\hline Maestro Ed. Infantil & 1304 & $96(7,36 \%)$ \\
Maestro Ed. Primaria & 2572 & $107(4,16 \%)$ \\
Total & 3876 & $203(5,23 \%)$ \\
\hline
\end{tabular}

educación preventiva del consumo de drogas, el período de formación inicial es clave por dos motivos: porque los jóvenes universitarios que son futuros docentes se encuentran en el intervalo de edad de mayor prevalencia de consumo de estas sustancias; porque los programas de formación deben enfocarse en el rechazo a las creencias erróneas sobre prevención a la que nos estamos refiriendo.

\section{Método}

\section{Objetivos}

Este estudio tiene dos objetivos: (1) conocer los hábitos de consumo de sustancias psicoactivas de los estudiantes de los maestros en formación inicial; (2) conocer el grado de acuerdo con las creencias erróneas más extendidas entre docentes sobre la educación para la prevención del consumo de sustancias psicoactivas; (3) conocer las correlaciones existentes entre hábitos de consumo y creencias educativas erróneas entre la población de referencia.

\section{Participantes}

La población de esta investigación está compuesta por los 3876 estudiantes de Magisterio de la Facultad de Ciencias de la Educación de la Universidad de Granada.

Tabla 3

La distribución por sexo, edad y especialidad de la Muestra.
De ellos, 1304 estudian el Grado de Magisterio en Educación Infantil, en tanto que 2572 estudian el Grado de Educación Primaria. La muestra la integran 203 estudiantes, el $5.23 \%$ de la población, como se muestra en la tabla 2, que fueron contactados, en su horario de clase, entre los grupos de estudiantes con actividad académica en el período de aplicación.

La distribución por sexo, edad y especialidad se presenta en la tabla 3. La muestra se distribuye en un $71.42 \%$ de mujeres y un $28.07 \%$ de varones; un $47.29 \%$ estudian el Grado de Educación Infantil en tanto que un $52.70 \%$ estudian el Grado de Educación Primaria. La edad de los participantes está en un intervalo de entre 17 y 30 años, siendo la media de 21 años.

\section{Instrumentos de evaluación}

Para la realización de este estudio se han aplicado dos instrumentos. El primero de ellos, con el que se cubre el objetivo de conocer los hábitos de consumo de la muestra, ha sido la encuesta elaborada y empleada por la Junta de Andalucía para sus estudios bienales " $L a$ Población Andaluza ante las Drogas", en su última versión (Junta de Andalucía, 2011), con la que se describe la prevalencia de sustancias psicoactivas entre la población andaluza y las características sociodemográficas de los consumidores. La encuesta consta de nueve dimensiones: consumo de tabaco y

\begin{tabular}{|c|c|c|c|}
\hline Sexo & & & Varón \\
\hline & & & $57(28.07 \%)$ \\
\hline Especialidad & & & $\begin{array}{c}\text { Educación Primaria } \\
107(52.70 \%)\end{array}$ \\
\hline Edad & $\begin{array}{c}\text { Min } \\
17\end{array}$ & $\begin{array}{c}\text { Max } \\
30\end{array}$ & $\begin{array}{c}\text { Media } \\
21\end{array}$ \\
\hline
\end{tabular}


Tabla 4

Consumo de tabaco

Consumo de tabaco

Nunca

55.7

Diariamente

alcohol; consumo de otras sustancias; botellón; conductas de riesgo; percepción del riesgo y la disponibilidad; información sobre drogas; actitud ante el consumo de drogas; prevención; y datos de situación personal y social. En la encuesta se indaga sobre patrones de consumo y edades de inicio en las distintas sustancias y permite establecer diferencias por sexo e intervalos de edad, con lo que la información que proporciona es muy amplia. Esta misma encuesta ha sido aplicada por nosotros a la muestra de estudiantes de Magisterio. El segundo instrumento aplicado a los sujetos es un inventario de creencias en escala tipo Likert de cuatro grados, confeccionado específicamente para el trabajo, a partir del listado de creencias erróneas sobre la educación preventiva del consumo de drogas elaborado por la Addiction Research Foundation ARF (1991), traducido por Bernardo Ruiz Victoria, e incluido en los recursos didácticos del Ministerio de Sanidad Servicios Sociales e Igualdad de España (Ruiz Victoria, n.d.) para la formación de profesores en la prevención del consumo de drogas, con el objetivos de conocer el grado de acuerdo de los estudiantes con cada una de ellas..

\section{Trabajo de campo y análisis de datos}

El trabajo de campo se realizó en el mes de octubre de 2015. La encuesta y el inventario de creencias se administraron de manera conjunta, y en ese orden, en una sesión única durante el horario de clase. Los sujetos pudieron realizarlo de manera voluntaria y anónima.

Los datos recogidos se han sometido a análisis estadísticos con el paquete estadístico SPSS V.20. Se

Tabla 5

Consumo de alcohol han realizado sendos análisis, descriptivo y un correlacional. El primero nos ha permitido establecer la media y la desviación típica de las variables contínuas, así como la frecuencia y el porcentaje de respuesta en las variables discretas. Con el segundo hemos establecido correlaciones bivariadas entre hábitos de consumo y creencias falsas, utilizando el coeficiente de Pearson.

\section{Resultados}

\section{Tabaco y alcohol}

Los estudiantes de magisterio presentan un nivel de consumo habitual o diario de tabaco del $17.2 \%$ (tabla 4 ), siendo más de la mitad de la muestra los que reconocen que nunca han consumido tabaco. Casi un tercio de la muestra admite haber consumido tabaco en alguna ocasión e incluso haber sido fumador en el pasado. En todo caso, como ya veremos, el porcentaje de consumidores diarios es sensiblemente inferior a las poblaciones andaluza y española de referencia.

El alcohol es, con diferencia, la sustancia psicoactiva con mayor prevalencia en la muestra. Hasta un $78.3 \%$ de los estudiantes admiten haber bebido en el último mes y hasta un $43.8 \%$ reconoce haberse emborrachado en los últimos 30 días (tabla 5). Los datos confirman la expansión del nuevo modelo de consumo de alcohol tipo atracón (binge drinking) asociado a la práctica social del botellón, pues hasta un $13.3 \%$ de los estudiantes reconocen practicarla todas las semanas.

\begin{tabular}{lccc}
\hline & $\begin{array}{c}\text { Consumo } \\
\text { (haber bebido en el } \\
\text { último mes) }\end{array}$ & $\begin{array}{c}\text { Consumo excesivo (haberse } \\
\text { emborrachado en el último } \\
\text { mes) }\end{array}$ & $\begin{array}{c}\text { Consumo en botellón } \\
\text { todas las semanas }\end{array}$ \\
\hline Consumo de alcohol & 78.3 & 43.8 & 13.3 \\
\hline
\end{tabular}


Tabla 6

Experiencia en consumo de sustancias

\begin{tabular}{lc}
\hline \multicolumn{1}{c}{ Experiencia en consumo de sustancias } & Porcentaje \\
\hline Marihuana & 41.4 \\
Hachís & 22.3 \\
Nuevas sustancias & 21.7 \\
Tranquilizantes con prescripción & 12.8 \\
Tranquilizantes sin prescripción & 5.9 \\
MDMA & 4.4 \\
Anfetaminas & 3.4 \\
Cocaína & 3.0 \\
Alucinógenos & 2.5 \\
Hipnóticos y somníferos con prescripción & 2.0 \\
Inhalables & 1.5 \\
Hipnóticos y somníferos sin prescripción & 1.0 \\
Éxtasis & 0.5 \\
Heroína & 0.5 \\
\hline
\end{tabular}

\section{Otras drogas}

Fuera del alcohol, sin duda es el cánnabis la droga más consumida por los estudiantes. Un $41.4 \%$ es o ha sido consumidor de Marihuana y hasta un $22.3 \%$ lo es o ha sido de Hachís. En la tabla 6 se recogen en orden de prevalencia las demás sustancias psicoactivas de cuyo consumo tienen experiencia los estudiantes de Magisterio. Tras el cánnabis aparecen las nuevas sustancias adictivas que imitan el efecto de las drogas ilegales y son conocidas como legal highs. Hasta un $21.7 \%$ de la muestra admite haber probado estas nuevas sustancias. Tiene una alta prevalencia el consumo de tranquilizantes bajo prescripción médica $(12.8 \%)$ y sin prescripción $(5.9 \%)$. Le siguen, en orden de prevalencia, el consumo de MDMA (4.4\%), Anfetaminas (3.4\%), Cocaína

\section{Tabla 7}

Regulación de la Producción, distribución y Venta de Cannabis

\begin{tabular}{|c|c|}
\hline \multicolumn{2}{|c|}{$\begin{array}{c}\text { Indica en qué medida estarías en acuerdo o en desacuerdo con la regulación de la producción, distribución y } \\
\text { venta de Cannabis (Hachís o Marihuana) }\end{array}$} \\
\hline & Porcentaje \\
\hline Muy de acuerdo & 22.2 \\
\hline De acuerdo & 21.7 \\
\hline Ni de acuerdo ni en desacuerdo & 22.2 \\
\hline En desacuerdo & 13.3 \\
\hline Muy en desacuerdo & 20.7 \\
\hline
\end{tabular}

(3.0\%), Alucinógenos (2.5\%), Hipnóticos y somníferos ( $2.0 \%$ con prescripción y $1.0 \%$ sin prescripción) e Inhalables $(1.5 \%)$. Por debajo del $1 \%$ se señala la experiencia en consumo de Éxtasis y Heroína.

\section{Opiniones sobre la legalización de las drogas}

En la tabla 7 aparecen los datos referidos a la legalización del Cánnabis. Las opiniones se reparten de manera muy proporcionada entre los diferentes grados de acuerdo, aunque podemos destacar que un $43.9 \%$ de estudiantes se declaran de acuerdo o muy de acuerdo, en tanto que sólo un $34 \%$ lo hace en desacuerdo o muy en 
Tabla 8

Regulación de la producción, distribución y venta de todas las drogas

\begin{tabular}{lc}
\hline \multicolumn{2}{c}{$\begin{array}{c}\text { Indica en qué medida estarías en acuerdo o en desacuerdo con la regulación de la producción, distribución y } \\
\text { venta de todas las drogas }\end{array}$} \\
\hline Muy de acuerdo & Porcentaje \\
De acuerdo & 20.7 \\
Ni de acuerdo ni en desacuerdo & 11.8 \\
En desacuerdo & 11.3 \\
Muy en desacuerdo & 16.7 \\
\hline
\end{tabular}

desacuerdo. Esto es, hay casi 14 puntos de diferencia a favor del acuerdo con posibles medidas de legalización y normalización del consumo de Cánnabis.

Respecto al resto de drogas, se presenta una situación inversa (tabla 8). Más de la mitad de la muestra se declara en contra de la regulación de su producción, distribución y venta $(56.1 \%)$, en tanto que un tercio de los encuestados $(32.5 \%)$ se muestran de acuerdo o muy de acuerdo con la medida, lo que significa más de 23 puntos de diferencia.

Tabla 9

Creencias erróneas sobre la Educación Preventiva

\section{Acuerdo con las creencias erróneas sobre la educación preventiva}

El inventario de creencias suministrado nos ha permitido conocer el grado de acuerdo o desacuerdo de los estudiantes de Magisterio con las principales creencias erróneas extendidas entre los profesionales de la enseñanza y señaladas por la ARF (1991). En la tabla 9 se muestran los datos obtenidos y con indicación de la media y el porcentaje de respuesta a cada una de las 4 op-

\begin{tabular}{|c|c|c|c|c|c|}
\hline Creencia & Media & 1 & 2 & 3 & 4 \\
\hline $\begin{array}{l}\text { Si beber o fumar es aceptable para los adultos, debería serlo también para los } \\
\text { niños }\end{array}$ & 1.34 & 72.4 & 23.2 & 2.5 & 2.0 \\
\hline $\begin{array}{l}\text { No se puede ayudar a los consumidores abusivos de alcohol u otras drogas } \\
\text { hasta que "toquen fondo" }\end{array}$ & 1.39 & 68.5 & 26.1 & 3.4 & 2.0 \\
\hline Mientras un niño use solo "drogas blandas", como la Marihuana, o solamente & & & & & \\
\hline $\begin{array}{l}\text { Alcohol y Tabaco, es muy difícil que llegue a ser adicto, y por lo tanto no } \\
\text { necesitará ningún tipo de intervención }\end{array}$ & 1.44 & 63.5 & 29.6 & 5.9 & 1.0 \\
\hline $\begin{array}{l}\text { Los alumnos son demasiado jóvenes para "engancharse", se necesitan años } \\
\text { para convertirse en adicto }\end{array}$ & 1.51 & 54.2 & 41.4 & 3.4 & 1.0 \\
\hline Lo que hacen los niños fuera de clase no es asunto de la escuela & 1.66 & 50.2 & 35.5 & 12.3 & 2.0 \\
\hline $\begin{array}{l}\text { La educación sobre drogas aumentará la curiosidad de los estudiantes por } \\
\text { probar las sustancias }\end{array}$ & 1.81 & 31.5 & 57.6 & 8.9 & 2.0 \\
\hline Es mejor beber alcohol que consumir drogas & 2.20 & 19.7 & 45.3 & 30.0 & 4.9 \\
\hline La educación sobre drogas es responsabilidad de la familia & 2.20 & 17.7 & 47.3 & 32.0 & 3.0 \\
\hline Sólo los especialistas pueden impartir educación sobre drogas & 2.28 & 15.3 & 40.9 & 30.0 & 13.8 \\
\hline $\begin{array}{l}\text { Los únicos que deben ayudar a los estudiantes con problemas con el alcohol } \\
\text { u otras drogas son los especialistas en adicciones }\end{array}$ & 2.42 & 11.3 & 56.7 & 24.6 & 7.4 \\
\hline $\begin{array}{l}\text { Las personas más apropiadas para hablar de los peligros del alcohol u otras } \\
\text { drogas a los estudiantes son ex-alcohólicos o ex-drogadictos }\end{array}$ & 2.59 & 6.9 & 36.5 & 47.8 & 8.9 \\
\hline
\end{tabular}


ciones ( 1 muy en desacuerdo; 2 en desacuerdo; 3 de acuerdo; 4 muy de acuerdo). En la tabla hemos ordenado las creencias erróneas en función del valor del mayor desacuerdo expresado por los estudiantes y, por tanto, de su rechazo expresado en la media alcanzada. Hemos constatado que, excepto una creencia, el resto es rechazado por la mayoría de los estudiantes, aun cuando se puede encontrar en todas ellas un porcentaje de entre 4.5 y 56.7 de la población que considera acertadas alguna de las creencias.

Las creencias que ha considerado acertadamente erróneas la mayoría de los encuestados son las referidas a que si beber o fumar es aceptable para los adultos, debería serlo también para los niños; no se puede ayudar a los consumidores abusivos de alcohol u otras drogas hasta que "toquen fondo"; mientras un niño use solo "drogas blandas", como la Marihuana, o solamente Alcohol y Tabaco, es muy dificil que llegue a ser adicto, y por lo tanto no necesitará ningún tipo de intervención; los alumnos son demasiado jóvenes para "engancharse", se necesitan años para convertirse en adicto; lo que hacen los niños fuera de clase no es asunto de la escuela; la educación sobre drogas aumentará la curiosidad de los estudiantes por probar las sustancias; es mejor beber alcohol que consumir drogas; La educación sobre drogas es responsabilidad de la familia; Sólo los especialistas pueden impartir educación sobre drogas; y los únicos que deben ayudar a los estudiantes con problemas con el alcohol u otras drogas son los especialistas en adicciones;

Por el contrario, la única creencia errónea que para una la mayoría de la muestra se considera erróneamente cierta (56.7 \%) es que las personas más apropiadas para

Tabla 10

Datos comparativos de Sustancias Psicoactivas hablar de los peligros del alcohol u otras drogas a los estudiantes son ex-alcohólicos o ex-drogadictos.

\section{Correlaciones entre consumidores, consumo de drogas y creencias erróneas}

Para cubrir el segundo objetivo de nuestro estudio, hemos analizado las correlaciones entre alguna característica del consumidor, el sexo, la experiencia en consumo de sustancias psicoactivas y las creencias erróneas sobre la educación escolar en drogas. En la tabla 10 se presentan los datos obtenidos. Hemos encontrado una correlación positiva con cuatro creencias erróneas con distinto nivel de significatividad.

Al nivel de .01 se correlaciona la creencia de que no se puede ayudar a los consumidores abusivos de alcohol u otras drogas hasta que "toquen fondo", con el sexo. Las mujeres de la muestran están significativamente más en desacuerdo con esta creencia. Igualmente, la creencia de que mientras un niño use solo "drogas blandas", como la marihuana, o solamente alcohol y tabaco, es muy dificil que llegue a ser adicto, y por lo tanto no necesitará ningún tipo de intervención, con el sexo, en el mismo sentido que la anterior. Las mujeres de la muestran están significativamente más en desacuerdo con esta creencia. Por último, a este nivel de significatividad, la creencia de que lo que hacen los niños fuera de clase no es asunto de la escuela, que correlaciona con el sexo, mujeres más en desacuerdo, y con consumo de tranquilizantes con prescripción, en el sentido de que los consumidores están más en desacuerdo con esta creencia errónea.

\begin{tabular}{lcccc}
\hline \multicolumn{1}{c}{ Sustancias psicoactivas } & Andalucía & España & $\begin{array}{c}\text { Estudiantes } \\
\text { Magisterio UGR }\end{array}$ & $\begin{array}{c}\text { Profesionales Salud } \\
\text { Andalucía }\end{array}$ \\
\hline Tabaco (diario) & 34.4 & 30.8 & 17.2 & 17.3 \\
Alcohol (último mes) & 67.3 & 64.4 & 78.3 & 64.0 \\
Cánnabis & 42.4 & 9.2 & 63.7 & 73.0 \\
Hipnosedantes & 11.9 & 22.2 & 18.7 & 23.0 \\
Drogas de diseño & 6.9 & 1.6 & 9.8 & 5.8 \\
Cocaína & 5.6 & 2.2 & 3.0 & 4.2 \\
Heroína & 0.4 & 0.1 & 0.5 & 2.5 \\
\hline
\end{tabular}


Al nivel de .05 se correlaciona la creencia de que no se puede ayudar a los consumidores abusivos de alcohol u otras drogas hasta que "toquen fondo", con el consumo de tranquilizantes con prescripción. Los consumidores están más en desacuerdo. La creencia de que mientras un niño use solo "drogas blandas", como la Marihuana, o solamente Alcohol y Tabaco, es muy dificil que llegue a ser adicto, y por lo tanto no necesitará ningún tipo de intervención, correlaciona con consumo de tabaco, consumo de alcohol y consumo de tranquilizantes sin prescripción. En todos los casos son los consumidores quienes están más en desacuerdo con la afirmación. Igualmente se correlaciona la creencia de que sólo los especialistas pueden impartir educación sobre drogas, con el consumo de tranquilizantes con prescripción en el sentido de que los consumidores están más en desacuerdo con ella.

\section{Discusión}

\section{Comparación de patrones de consumo}

El patrón de consumo de los estudiantes de Magisterio está, en parte, ajustado a los patrones de consumo establecidos entre la población andaluza (Junta de Andalucía, 2011) y la población española (MSSI, 2015). En la tabla 6 se recogen estos datos comparados y se incluyen los datos sobre consumo de sustancias psicoactivas recogidos por Juárez Jiménez (2015) para profesionales de la salud. Nuestra intención es poder remitir nuestras evidencias a poblaciones de referencia, así como comparar los patrones de consumo de los futuros profesionales de la enseñanza, con otro colectivo profesional de máxima importancia en la educación para la prevención de adicciones como es el de los profesionales de la salud.

El consumo diario de tabaco es similar entre los futuros profesionales de la enseñanza $(17.2 \%)$ y los profesionales de la salud (17.3\%), representando la mitad de las tasas de consumo en Andalucía (34.4\%) y en España (30.8\%). Sin embargo, el consumo de alcohol de los estudiantes de Magisterio se eleva sensiblemente, entre 11 y 14 puntos, sobre las tasas de Andalucía (67.3\%), de España $(64.4 \%)$ y de los profesionales de la salud $(64.0 \%)$. Observamos una gran disparidad en los datos sobre consumo de Cánnabis, pero en todo caso, el consumo de los estudiantes de Magisterio (63.7\%) es superior a la media andaluza (42.4\%) y a la media española (9.2\%), aunque inferior a la tasa de consumo en profesionales de la salud $(73.0 \%)$. El consumo de hipnosedantes de nuestra muestra (18.7\%) es nuevamente superior a las medias andaluza (11.9\%) y española $(22.2 \%)$, aunque inferior a la muestra de profesionales de la salud (23.0\%). Los estudiantes de Magisterio presentan la tasa mayor de consumo de drogas de diseño $(9.8 \%)$, seguidos de la población andaluza $(6.9 \%)$, del colectivo de profesionales de la salud (5.8\%), y de la tasa de referencia entre la población española $(1.6 \%)$. El consumo reconocido de Cocaína de los estudiantes de Magisterio (3.0), se acerca al de referencia de la población española $(2.2 \%)$ y es inferior al de los profesionales de la salud $(4.2 \%)$ y al de la población andaluza $(5.6 \%)$. Por último, el consumo de heroína $(0.5 \%)$ es superior al de las poblaciones andaluza $(0.4 \%)$ y española $(0.1 \%)$ de referencia, e inferior al consumo manifestado por los profesionales de la salud $(2.5 \%)$.

En todo caso, la comparación del patrón de consumo de los estudiantes de Magisterio con las poblaciones andaluza y española, está limitada tanto por el intervalo de edad al que corresponden los datos como por la fecha de los propios datos y el período de tiempo respecto al que se realizan curvas de evolución.

Los datos de la población andaluza corresponden al año 2011 y la evolución que se observa en el consumo de las distintas sustancias entre los años 1994 a 2011, es muy variada. Los datos disponibles de la población española son de 2015 e incluyen curvas de evolución desde 1997. En estos períodos de tiempo se observa un ligero repunte del consumo de tabaco en Andalucía, en tanto se mantiene prácticamente estable entre la población española en todos los intervalos de edad. Se 
observa también un repunte del consumo de alcohol en Andalucía, aun cuando cambia el patrón de consumo, disminuyendo el modelo de consumo diario por el modelo de consumo de atracón de fin de semana. El consumo de alcohol se mantiene estable en el tiempo entre la población española en niveles alarmantemente altos. En este período se eleva claramente el consumo de Cánnabis y nuevas sustancias, en tanto que se mantiene o reduce el consumo de las otras drogas entre la población andaluza y entre la población española.

El intervalo de edad al que corresponden la mayoría de los estudiantes de Magisterio, 17 a 24 años, se corresponde con los intervalos de edad donde aparecen más consumidores de sustancias psicoactivas entre la población andaluza y la población española.

Discusión aparte merece la comparación entre los estudiantes de Magisterio y los profesionales de la salud. Se observa un menor consumo de tabaco y un mayor consumo de alcohol, tipo atracón, entre los primeros respecto a los segundos, lo que puede estar relacionado con prevalencia de la práctica del botellón asociada más extendida entre jóvenes universitarios que entre profesionales de mayor edad. Respecto al consumo de otras drogas, aparece una mayor prevalencia entre profesionales de la salud, excepto en el consumo de drogas de diseño, lo que puede estar, nuevamente asociada a la diferencia de edad entre ambos colectivos.

\section{Opiniones y creencias sobre las drogas}

El estudio ha demostrado que en la nueva generación de jóvenes que se forman como maestros, son mayoría los que están de acuerdo con la legalización de las denominadas drogas blandas como el cánnabis. Entendemos que esta posición está relacionada con la amplia prevalencia de consumo que se observa en la población.

El estudio ha demostrado que esta generación de jóvenes que se forman para maestros, realiza una mejor discriminación de las creencias erróneas en educación preventiva, que la que fue descrita en su momento por la
ARF en 1991. Ello no quiere decir que todas las creencias erróneas estén desterradas, ni que existan grupos de población minoritarios que aún las mantienen. En la identificación de esos grupos deben centrarse los esfuerzos que se realicen en formación inicial para desterrar de manera definitiva estas creencias y lograr mayor eficacia en los programas preventivos (Longshore, Ellickson, McCaffrey y St Clair, 2007).

\section{Conclusiones}

Con este estudio se ha permitido establecer un patrón de hábitos de consumo de sustancias psicoactivas de los estudiantes de Magisterio de la Universidad de Granada. El patrón de consumo establecido es coherente con las referencias de la población andaluza y española ajustado a los intervalos de edad correspondiente. Por tanto, concluimos que los estudiantes de Magisterio, que van a ser responsables de la ejecución de programas de prevención del consumo de sustancias psicoactivas, no presentan hábitos distintos de consumo al otro colectivo de profesionales implicados en la educación preventiva, los profesionales de la salud, ni al resto de la población. Es importante que se realice un seguimiento de los hábitos de consumo de este colectivo y que se puedan comparar en el futuro con los hábitos de consumo del profesorado en ejercicio tanto de los docentes de Educación Infantil y Primaria como de los docentes de Educación Secundaria en quiénes recae la responsabilidad de asumir el liderazgo pedagógico del desarrollo de programas de educación para la salud y el desarrollo de hábitos saludables, en general, y la realización de planes de prevención del consumo de sustancias psicoactivas, en concreto. El estudio nos ha permitido realizar una primera aproximación a las opiniones y creencias sobre la educación preventiva que mantienen los futuros docentes. Concluimos que, en general, las falsas creencias sobre educación preventiva son rechazadas por los estudiantes de magisterio. Ahora bien, el estudio permite señalar grupos de estudiantes que aún conservan las falsas creencias. Por ello es importante que, en las acciones de formación docente sobre drogas, en formación inicial, se considere el rechazo general de las creencias erróneas que dificultan la prevención en la escuela. 


\section{Referencias}

Adams, M. L., Jason, L. A., Pokorny, S. y Hunt, Y. (2009). The relationship between school policies and youth tobacco use. Journal of School Health, 79(1), 17-23. http://dx.doi.org/10.1111/j.17461561.2008.00369.x

American Psychological Association. (2008). Are zero tolerance policies effective in the schools? American Psychologist, 63(9), 852-862. http://dx.doi.org/10.1037/0003-066X.63.9.852

Addiction Research Foundation ARF (1991). Teacher Training in Prevention. Meeting the Challenge of Alcohol and Other Drugs. Ontario, Canadá: Addiction Research Foundation.

Bryant, A. L. y Zimmerman, M. A. (2002). Examining the effects of academic beliefs and behaviors on changes in substance use among urban adolescents. Journal of Educational Psychology, 94(3), 621-637. http://dx.doi.org/10.1037/0022-0663.94.3.621

Burrow-Sanchez, J. J. y Lopez, A. L. (2009). Identifying substance abuse issues in high schools: A national survey of high school counselors. Journal of Counseling and Development, 87(1), 72-79. http://dx.doi.org/10.1002/j.1556-

6678.2009.tb00551.x

Burrow-Sanchez, J., Call, M. E., Adolphson, S. L. y Hawken, L. S. (2009). School psychologists' perceived competence and training needs for student substance abuse. Journal of School Health, 79(6), 269-276. http://dx.doi.org/10.1111/j.17461561.2009.00409.x.

CASA (2011). The National Center on Addiction and Substance Abuse at Columbia University. Adolescent Substance Use: America's \#1 Public Health Problem. New York: CASA.

Córdoba, R., Cabezas, C., Camarelles, F., Gómez, J., Damián, D., López A., ... y Ramírez J. I. (2012). Recomendaciones sobre el estilo de vida. Atención
Primaria, 44(1), 16-22.

http://dx.doi.org/10.1016/S0212-6567(14)70048-4

Cox, R. G., Zhang, L., Johnson, W. D. y Bender, D. R. (2007). Academic performance and substance use: Findings from a state survey of public high school students. Journal of School Health, 77(3), 109-115. http://dx.doi.org/10.1111/j.1746-1561.2007.00179.x

Dawkins, M. P., Wiliams, M. M. y Guilbault, M. (2006). Participation in school sports: Risk or protective factors for drug use among black and white students? Journal of Negro Education, 75 (1), 2533.

Engberg, J. y Morral, A. R. (2006). Reducing substance use improves adolescents' school attendance. Addiction, 101(12), 1741-1751. http://dx.doi.org/10.1111/j.1360-0443.2006.01544.x

Fernández Cruz, M. (2015). Formación y desarrollo de los profesionales de la educación. Un enfoque profundo. Wisconsin MA: Deep University Press.

Harrison, P. A. y Narayan, G. (2003). Differences in behavior, psychological factors, and environmental factors associated with participation in school sports and other activities in adolescence. Journal of School Health, 73(3), 113-120. http://dx.doi.org/10.1111/j.17461561.2003.tb03585.x

Hoffmann, J. P. (2006). Extracurricular activities, athletic participation, and adolescent alcohol use: Genderdifferentiated and school-contextual effects. Journal of Health and Social Behavior, 47 (3), 275-290. http://dx.doi.org/10.1177/002214650604700306

Juárez Jiménez, M. V. (2015). Consumo de alcohol, tabaco, drogas ilegales de los profesionales internos residentes de Andalucía y el consejo clínico (tesis doctoral). Universidad de Jaén.

Junta de Andalucía (2011). La población andaluza ante las drogas XII. Sevilla, España: Consejería de Salud y Bienestar Social de la Junta de Andalucía. 
LaRusso, M. D., Romer, D. y Selman, R. L. (2008). Teachers as builders of respectful school climates: Implications for adolescent drug use norms and depressive symptoms in high school. Journal of Adolescence, 37(4), 386-398. http://dx.doi.org/10.1007/s10964-007-9212-4

Longshore, D., Ellickson, P. L., McCaffrey, D. F. y St Clair, P. A. (2007). School-based drug prevention among at-risk adolescents: Effects of ALERT plus. Health Education and Behavior, 34 (4), 651-668. http://dx.doi.org/10.1177/1090198106294895

MSSI (2015). Encuesta sobre alcohol y drogas en España. Informe EDADES 2015. Ministerio de Sanidad, Servicios Sociales e Igualdad.

ONU (2002). Manual sobre programas de prevención del uso indebido de drogas con participación de los jóvenes. Una guía de desarrollo y perfeccionamiento. New York: Oficina de las Naciones Unidas de Fiscalización de Drogas y Prevención del Delito de Viena.

Pisetsky, E. M., Chao, Y. M., Dierker, L. C., May, A. M. y Striegel-Moore, R. H. (2008). Disordered eating and substance use in high-school students: Results from the Youth Risk Behavior Surveillance System. International Journal of Eating Disorders, 41 (5), 464-470. http://dx.doi.org/10.1002/eat.20520.

Ringel, J. S., Ellickson, P. L. y Collins, R. L. (2007). High school drug use predicts job-related outcomes at age 29. Addictive Behaviors, 32 (3), 576-589. http://dx.doi.org/10.2975/35.3.2012.235.243

Ruiz Victoria, B. (n.d.). Guía del profesorado. El reto del alcohol y las drogas en la escuela. Madrid: Ministerio de Sanidad, Servicios Sociales e Igualdad [Documento web]. Recuperado de http://www.lasdrogas.info/guias-profesorado.html. 